\title{
Novel Fuzzy Association Rule Image Mining Algorithm for Medical Decision Support System
}

\author{
P. Rajendran \\ Department of CSE \\ K. S. Rangasamy College of Technology \\ Tiruchengode- 637215 \\ India.
}

\author{
M. Madheswaran \\ Center for Advance Research, Department of \\ Electronics and Communication Engineering \\ Muthayammal Engineering College \\ Rasipuram - 637408, India
}

\begin{abstract}
The proposed method deals with the detection of brain tumor in the CT scan brain images. The preprocessing technique applied on the images eliminates the inconsistent data from the CT scan brain images. Then feature extraction process is applied to extract the features from the brain images. A Novel Fuzzy Association Rule Mining (NFARM) applied on the image transaction database which contains the features that are extracted from the CT scan brain images. A new test image has been tested with the mined (NFARM) rules. The proposed NFARM gives the diagnosis keywords to physicians for making a better diagnosis system. The experimental results of the proposed method gives better performance compared to the traditional Fuzzy Apriori algorithm.
\end{abstract}

\section{Categories and Subject Descriptors}

MATLAB 7.0, Feature extraction, Pixel Intensity, Gray Level, Regions of Interest

\section{General Terms}

Progressive Water Immersion Algorithm, Fuzzy Partitioning Algorithm, Performance, Efficiency, Support, Rules and Formula

\section{Keywords}

Novel Fuzzy Association Rule Mining (NFARM), classification, Pre processing, Feature Extraction, medical imaging, image mining, data mining

\section{INTRODUCTION}

Brain tumor is one of the most common cause of cancer related deaths amongst the human being in the world and also the most frequently diagnosed form of cancer. Detecting brain tumor in it earliest stage is the key for its successful treatment.

For this earliest detection, a diagnosis method called the CTScan (Computerized Tomography Scan) is used. CT scan of brain images is one of the most popular, cost effective and relatively accurate method of early detection of the disease [1]. Any visual sign of abnormalities are carefully searched by the neuroradiologist from each image of CT scan. However the abnormalities are some time not prominent and often embedded because of the varying densities of cancer tissue structure. But during the routine screening, estimates indicate that between 10 and $30 \%$ of tumor cells are missed by the radiologist. Thus a variety of computer aided diagnosis (CAD) has been proposed to increase the efficiency and effectiveness of screening procedures[2]. The CAD system assists the physicians, as a "second option" by indicating the location of suspicious abnormalities in CT scan brain images.

The proposed method (NFARM) classifies the brain CT images into three categories: Normal, Benign and Malignant. Normal images characterizes the healthy patient, Begnin images represent a cancerous cells but not actually a tumor, and the third is Malignant images which are the actual form of tumor cells in patients.

Fuzzy Association Rule uses fuzzy logic to convert numerical attributes to fuzzy attributes thus maintaining the integrity of the information conveyed by such numerical attributes [3][4][5][11]. The popular fuzzy association rule mining algorithms that are available today are fuzzy apriori and its different variations [10]. Fuzzy apriori algorithm has been well supported for the numerical analysis[9], but where as in medical image classifications [18] it is less efficient.

The proposed method based on Novel Fuzzy Association Rule Mining helps in diagnosing the knowledge pattern from the medical images of CT scan image database. This proposed method has the advantage of selecting only the most relevant features to be used during the mining process [6][7]. Moreover this approach is optimized because it performs two operations in a single step that is feature selection and discretization [8]. This makes the mining algorithm faster, and also improves the classification accuracy. The overall view of the proposed system is given in the figure 1.1.

The proposed system consists of, two phases they are: 1 . Training Phase and 2. Testing Phase. In the training phase the images from the CT scan are pre processed and regions of interest are found. This ROI contains the objects holding their corresponding attributes are stored in the transactional database and NFARM is applied to mine the table of rules. In the testing phase the same process is repeated to find the rules, then the comparison is made to detect the brain tumor is diagnosed. 


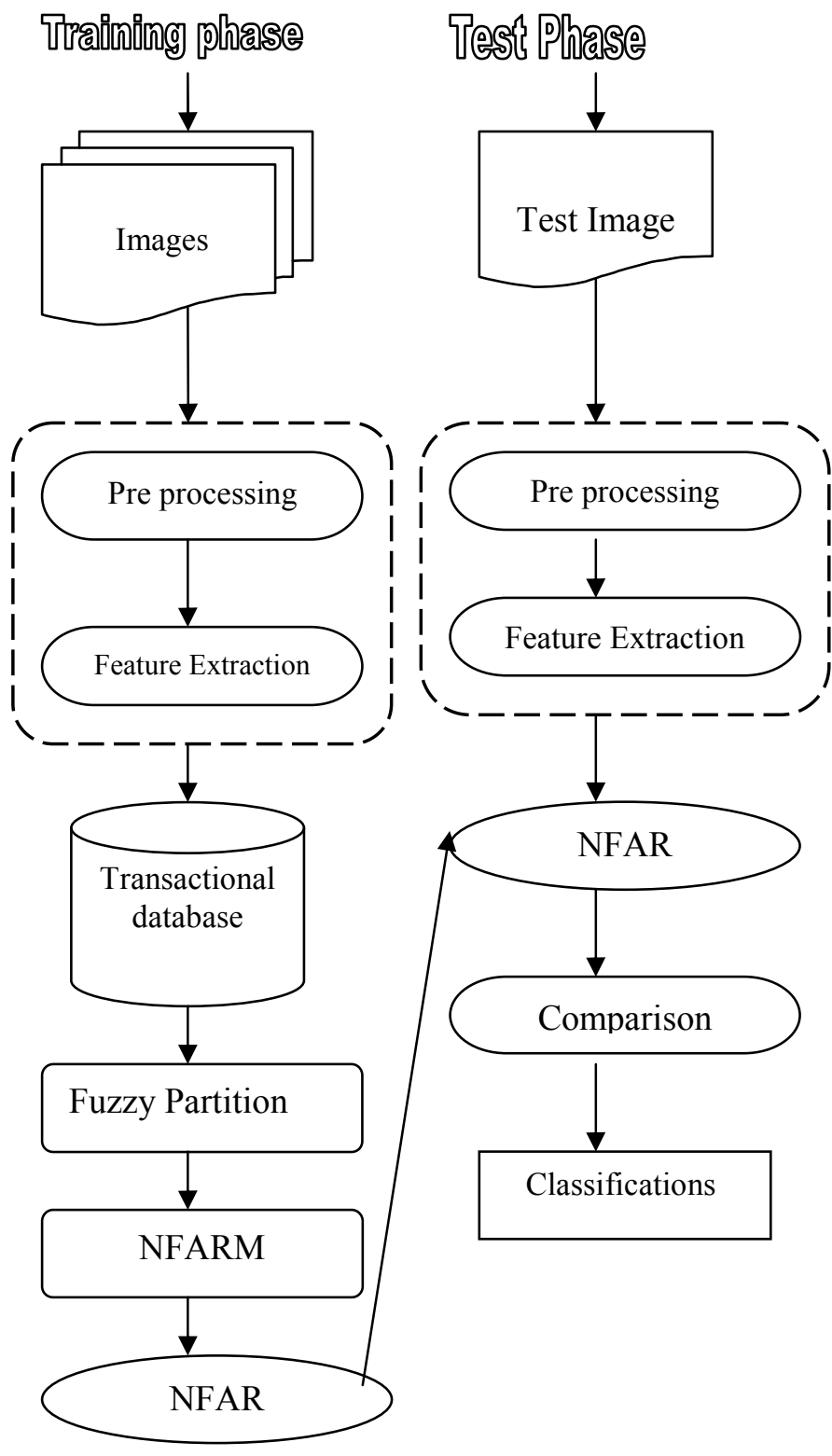

Figure 1.1 overview of the proposed system

In this paper, section 2 gives the related works, section 3 describes about the System description which includes pre processing and feature extraction techniques, section 4. Section deals about the Novel Fuzzy Association Rule and Fuzzy Measures, Section 5. Represents experimental results Section 6. Describes the conclusion and the last Section 7. Describes with the references.

\section{RELATED WORKS}

In paper [19] The concepts of data mining is described which includes the different types of basic algorithms used for mining all explained briefly. It also includes the most popularly used Association rule mining algorithm called Apriori algorithm and its basics. In paper [6] Association rule mining and efficiency in large database has been employed. In paper[20] the basic concepts of digital image processing applied in the field of Image mining and also the feature extraction techniques like gray level and histogram equalization are given. The details of regions of interest and also the algorithm used like watershed and Water immersion algorithm are given in detail. Most of the immersion algorithm are given in detail. Most of the fuzzy ARM work are directed towards theoretical aspects of finding, both positive and negative ones.[4]][5]. Fuzzy Apriori, the defacto algorithm used for fuzzy association rule mining is used in [7][11][12]. In order to crate fuzzy partitions from datasets, points that are belonging to different clusters are given in [10]. A Multimedia data mining system prototype multimedia miner [13][14] uses a data cube structure for mining characteristic, association and classification rules. Discovering association rules algorithm based on image content from a simple image dataset is presented. Research in image mining can be broadly classified into two main directions [17]. The first direction involves domain-specific applications.

\section{SYSTEM DESCRIPTION}

The proposed system consists of training phase and testing phase. In both the phases the pre processing and feature extraction techniques have been followed. They are detailed in the following subsections.

\subsection{Pre-processing}

Pre processing mines the actual data by eliminating the noise, inconsistent and incomplete data. It significantly improves the effectiveness of the mining technique[16]. As the CT-scan brain images appears for bright and for dark at different illumination conditions, the images are difficult to interpret, pre-processing is necessary to improve the quality of images and makes the feature extraction technique more reliable.

There are two reasons for using the CT scan brain images,

- The Scanner images contain anatomical information which offers the possibility to plan the direction and the entry points of the radiotherapy rays which have to target the cancerous cells and to avoid some risk organs.

- By using rays the CT Scan images obtained, all the same as the physical principle as radiotherapy. Since the radiotherapy rays intensity can be computed from the scanner images, the above said two reasons are very important 


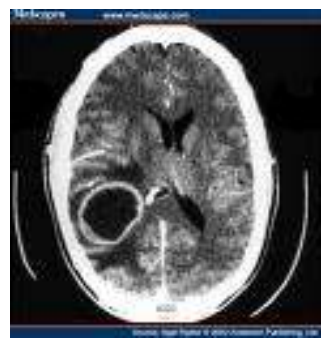

Figure 3.1 The original gray scaled brain image produced by CT scan

Figure 3.1 describes the original image produced by the CT scan. Use of Water immersion algorithm is a powerful technique for ROI detection works by grouping pixels with similar gradient information. But using this technique over segmentation of the trivial regions can be produced by digitizing the medical images. Instead, in this paper a progressive water immersion technique with domain knowledge guidance is used. This technique will detect the regions of interest (ROI) in medical images. A mining information table cab be formed by combining the ROI with the location, Size and other descriptors.

In the progressive water immersion algorithm the local optimal points in the images are located using the sliding window concept. This method will use a N X N to locate the local optimal points. The center of the window can be placed over each pixel in patches for each segmented patches. The central pixel inside the window will become local optimum when the gray level of the central pixel is optimal with respect to all other pixels in the window. Otherwise, in search for all local optimal points, the window will move to be centered at another pixel. Hence all the optimal points are matched at the end of this phase and they will be treated as the starting seeds for this technique. The main advantage of using the sliding window menthod is that it is possible to eliminate a large amount of optimal points that correspond to the light $\&$ dark reflection regions with the appropriate window size and hence ti removes the possibility of false detection.

This is because the light and dark reflection patches are generally lower and higher than that of potential ROI corresponding to the gray level. The distance between the optimal points in the patches and the nearest optimal points of the neighboring ROI are generally less than that between two touching ROI is defined. The window size can be set in such a way that these false optimal points are "absorbed" by the neighboring ROI optimal points while the true optimal points are not affected.

The water immersion process starts from the detected points and progressively floods its neighboring pixels, that have identified the true optimal points. There are 8 directions defined for the neighboring pixels. These neighbors are placed in a growing queue structure and are sorted in descending order of the gray level of the pixels. In the growing queue the lowest and highest pixel will be "immersed" first. And respectively all the other gray pixels are immersed. But the immersed pixels are marked as belonging to the same region label as the current seed.

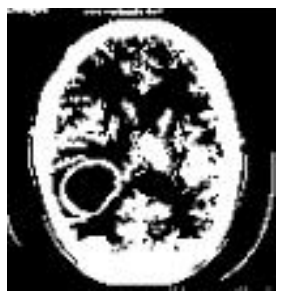

Figure 3.2. Darker and lighter reflection patches of potential ROI

Figure 3.2 illustrates the light and darker reflection patches. Then from the growing queue the matched pixels are removed. The immersion process continues until the growing queue is empty. The simple application of water immersion technique has the tendency of over flooding, To over come this problem, some rules are given here

Rule 3.1.1. We partition all pixels in pixel set $\mathrm{P}$ into $\mathrm{m}$ blocks. Pixels in the same block have the same gray level and pixels in the different blocks have the different gray level. Let $\mathrm{G}(\mathrm{P})=\{\mathrm{g} 1$, $\mathrm{g} 2, \ldots, \mathrm{gm}\}$ be P's gray-scale (GS) set if $\mathrm{G}(\mathrm{P})$ is an ascending sort set of g1', g2', ..., gm' and gi' is gray level of pixels in the ith block, where gi $(\mathrm{i}=1, \ldots, \mathrm{m})$ is the ith GS, g1' and gm' are P's minimum and maximum GS respectively. The GS of pixel pi is denoted as $\mathrm{g}(\mathrm{pi})$.

Rule 3.1.2. We call gmean( $\mathrm{P})$ the mean GS if

$$
\begin{aligned}
& |\mathrm{P}| \\
& \operatorname{gmean}(\mathrm{P})=\Sigma \mathrm{g}(\text { pi }) /|\mathrm{P}| \text {. } \\
& \mathrm{i}=1
\end{aligned}
$$

Rule 3.1.3. For any $\mathrm{P}$ and distance function $\mathrm{Dis} A=\mid \mathrm{gk}$ gmean $(\mathrm{P}) \mid$, mid-value GS is a middle value in the GS set that minimizes DisA. Mid-value GS set is a set of mid-value GS.

Rule 3.1.4. For any $\mathrm{P}$, if (1) Mid-value GS set includes one element gmid, and gs is the minimum value between gmean and gmid; (2) Mid GS set includes two elements, gs is the minimum value between these two values; gs is called Benchmark GS and another one is denoted as gs'.

Rule 3.1.5. For pixel set $P$, let

$\mathrm{g}^{(\mathrm{l})}=\{\mathrm{gi}|\mathrm{g} 1 \leq \mathrm{gi} \leq \mathrm{g} 1+| \mathrm{g} 1-\mathrm{gs} \mid / 2\}$ be low bound GS; $\mathrm{g}^{(\mathrm{h})}=\{\mathrm{gi} \mid \mathrm{gm}-$ $|\mathrm{gm}-\mathrm{gs}| / ,2 \leq \mathrm{gi} \leq \mathrm{gm}\}$ be high bound GS;

$\mathrm{g}^{(\mathrm{b})}=\mathrm{g}(\mathrm{l}) \mathrm{U} \mathrm{g}(\mathrm{h})$ be bound GS;

The progressive water immersion ignores all those pixels whose gray level doesn't belong to the bound GS.

Bound GS is defined with guidance of domain knowledge that describes the degree of dark and light. Figure 3.3 describes the optimality of point. So the optimality of point in a certain region is defined as follows, 
Optimality $=\left\{\begin{array}{l}\text { maximal grey level, if point belongs to high bound pixel set; } \\ \text { minimal grey level, if point belongs to low bound pixel set; }\end{array}\right.$

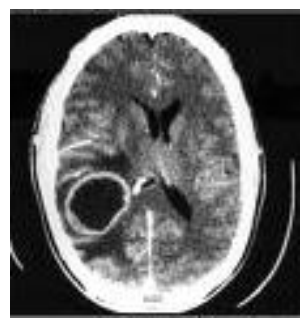

Figure 3.3. Optimal point in certain region

\subsection{Feature Extraction}

In medical image diagnosis, the earliest phase of a CAD system demands to extract the main image features regarding a specific criterion [21]. Essentially the most representative feature vary according to the image type (brain, mammograms or lung). By applying progressive water immersion algorithm, the images are segmented into objects [15]. Let $\mathrm{IM}=\left\{\mathrm{IM}_{1}, \mathrm{IM}_{2}, \ldots \ldots \mathrm{IM}_{\mathrm{N}}\right\}$ be a image set, after applying the algorithm, each image $\mathrm{IM}_{\mathrm{j}}$ will contain $k$ objects $R_{j 1}, R_{j 2}, \ldots . . R_{j K}$ For different images, the value of $K$ may not be same.Let $M$ be the total number of objects in IM, then the object set can be denoted as $R=\left\{R_{1}, R_{2}\right.$, ......... $\mathrm{R}_{\mathrm{M}} \quad$ Figure 3.4 describes the segmented images into objects.

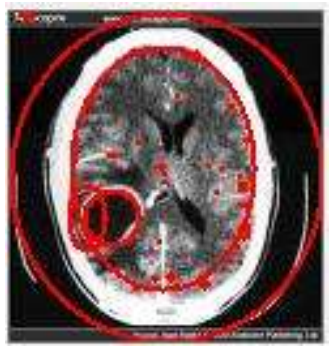

Figure 3.4. segmented image into objects

The relevant information for feature mining has to be extracted from object $\mathrm{R}_{\mathrm{i}}$. The distribution of density in the left hemisphere of the brain is almost identical with the right that is for normal persons the brain structure is nearly the same that is evident to be bilateral symmetry.

The different discriminative power to cluster object into different groups are provided here,

- Gray level of the object of interest

- Area of the object of interest

- Location of the object of interest
- Elongation of the object of interest

- Direction of the object of interest

- Symmetry of the object of interest

The objects that are extracted from brain images, with the above method is either brightness or darkness. Let the gray level is represented as GL. The value of brightness and darkness are equal to 0 and 1 respectively. The second feature is useful to relate the size of the region in the form of the area of the region.The area of ROI is defined as the total number of pixels within the region, up to and including the boundary pixels. The location of an object of interest is defined as a ratio. The formula given below are used to compute the coordinates of the centroid of the object,

$$
\bar{x}=\frac{1}{k} \sum_{j=1}^{k} x_{j} \quad \bar{y}=\frac{1}{k} \sum_{j=1}^{k} y_{j}
$$

$\mathrm{k}$ is the number of the pixels of the object and the location is $(x$ $/|\mathrm{x}|, y /|\mathrm{y}|)$.

The fourth feature is the elongation of an object which is defined as the ratio of the width of the minor axis to the length of the major axis. This ratio is computed as the minor axis width distance divided by the major axis length distance, giving a value between 0 and 1 .

Elongation of an object $=\frac{\text { minor axis width distance }}{\text { major axis length distance }}$

If the ratio is equal to 1 , the object is roughly a square or is circular in shape. As the ratio decreases from 1, the object becomes more elongated. Major axis is the longest line that can be drawn through the object. The two end points of the major axis are found by selecting the pairs of boundary pixels with the maximum distance between them. This maximum distance is also known as the major axis length. Similarly, the minor axis is defined as the line that it is perpendicular with respect to the major axis and the length between the two end points of the line intersected with the object is the longest which is called the width of the minor axis. The ratio, elongation, is a measure of the degree of elongation of an object.

elongation $=\operatorname{len}_{\text {major }} /$ len $_{\text {minor }}$

where, lenmajor is the major axis length of the object and lenminor is the minor axis length of the object. We establish a common co-ordinate with brain midline as y and perpendicular line going through the midpoint of midline as $\mathrm{x}$. With the major axis of the object, we define the next feature, direction of the object, as the inclination $\theta$ of the major axis and $x$ positive 
direction and $\theta \in[0,180]$. Figure 3.5 illustrates the location and size of the segmented image. Before introducing the final feature, we will give some following rules.

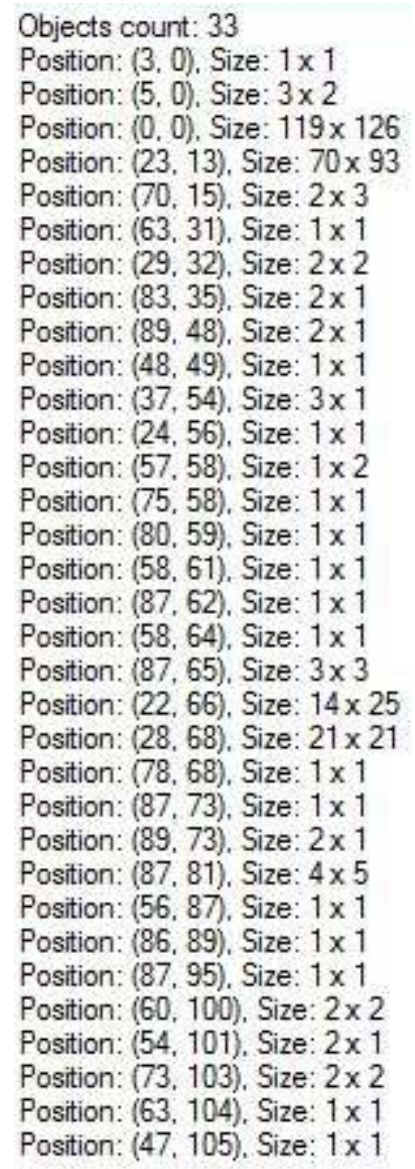

Figure 3.5. Location and Size of segmented portions

Rule 3.2.1. Pixel set of IMp is defined as $\mathrm{P}=\{$ pi|pi is the pixel with coordinate (xi, yi) in the image $\mathrm{IMp}\}, \mathrm{P}(\mathrm{L})$ and $\mathrm{P}(\mathrm{R})$ are pixel set of $\mathrm{IMp}(\mathrm{L})$ and $\mathrm{IMp}(\mathrm{R})$ respectively.

Rule 3.2.2. For any pli $\in \mathrm{P}(\mathrm{L})$, prj $\in \mathrm{P}(\mathrm{R})$, they are symmetric pixel if the line between pli and prj is halved vertically by brain midline. They are denoted as pli and pri below.

Rule 3.2.3. $\Delta \mathrm{g}(\mathrm{P})$ is IMp's difference set if for any symmetrical pixel pli and pri, $\Delta \mathrm{g}(\mathrm{P})=\{\Delta \mathrm{gi} \mid \Delta \mathrm{gi}=\mathrm{g}($ pli) $-\mathrm{g}($ pri $)$,

$\mathrm{i}=1,2, \ldots,|\mathrm{p}| / 2\}$, where $\mathrm{g}(\mathrm{pli})$ and $\mathrm{g}($ pri) are gray level of these two pixels respectively. Now we define the sixth feature as following.

After the above process, all the ROIs that are detected can be called as objects. Next, images with many different objects are represented by transactions, but there exist relationship among these objects and identical objects (either bright or dark) can repeat in an image. Therefore, we use a table to describe these transactions. In table 3.1, Part I and Part II is image id and object id respectively. Part III is the attribute set of object, for example, gray level, location, etc., and Part IV is the relationship predicate set, for instance, next-to, inside and predicate related to domain. Part V is the attribute set of image.

Table 3.1. Images are modeled by transactions

\begin{tabular}{|l|l|l|l|l|}
\hline Part I & Part II & Part III & Part IV & Part V \\
\hline $\begin{array}{c}\text { Image } \\
\text { id }\end{array}$ & $\begin{array}{c}\text { Object } \\
\text { id }\end{array}$ & Oattr1 & RP1 & Iattr1 \\
\hline $\mathrm{IM}_{1}$ & $\mathrm{O}_{1}$ & Oattr_V & $\mathrm{O}_{2}$ & Iattr $_{1}$ \\
\hline $\mathrm{IM}_{1}$ & $\mathrm{O}_{1}$ & $\cdots$ & $\cdots$ & $\cdots$ \\
\hline $\mathrm{IM}_{1}$ & $\mathrm{O}_{1}$ & $\cdots$ & $\cdots$ & $\cdots$ \\
\hline $\mathrm{IM}_{2}$ & $\mathrm{O}_{2}$ & $\cdots$ & $\cdots$ & $\cdots$ \\
\hline $\mathrm{IM}_{2}$ & $\mathrm{O}_{2}$ & $\cdots$ & $\cdots$ & $\cdots$ \\
\hline$\cdots$ & $\cdots$ & $\cdots$ & $\cdots$ & $\cdots$ \\
\hline $\mathrm{IM}_{\mathrm{n}}$ & $\mathrm{O}_{\mathrm{n}}$ & $\cdots$ & $\cdots$ & $\cdots$ \\
\hline
\end{tabular}

\section{NOVEL FUZZY ASSOCIATION RULES AND FUZZY MEASURES}

At the time of novel fuzzy ARM process, a number of fuzzy partition is defined on the image domain of each attribute [12]. Figure 4.1 describes about the fuzzy partition on image domain with different attribute values. As a result the extended attribute value is in the interval $[0,1]$ is transformed from the original datasets is the transactional database for forming the NFARM rules. In order to process this dataset, new measures are used in terms of t-norms. The generation of NFARM is directly impacted by the fuzzy measures.

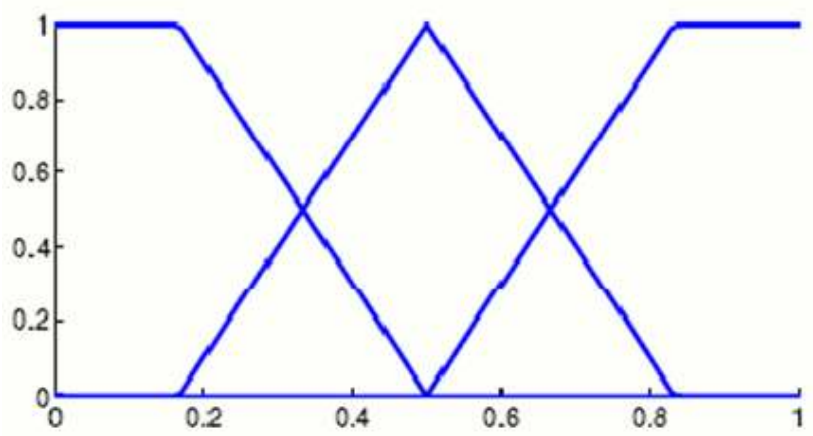

Figure 4.1 Fuzzy partitions on image domain of different attribute values

\subsection{Fuzzy Partitioning Algorithm}

Dataset $D=\left\{x_{1}, x_{2}, \ldots . x_{n}\right\}$ where $x_{1}, x_{2}, \ldots . x_{n}$ are different records set of quantitative attributes $\mathrm{QA}=\left\{\mathrm{q}_{1}, \mathrm{q}_{2}\right.$, $\left.\ldots . . \mathrm{q}_{\mathrm{r}}\right\}$ set of fuzzy partitions $\mathrm{FP}=\left\{\mathrm{FP}_{1}, \mathrm{FP}_{2} \ldots \ldots \ldots \mathrm{FP}_{\mathrm{S}}\right\}-$ Set of fuzzy partitions of quantitative attributes $\mathrm{q}_{\mathrm{m}}$ applied on the image set.

Given a dataset D which has both object and their corresponding attributes. Each attribute and the values for it are singled out. A 
fuzzy partition is obtained by using the ROI containing its corresponding attribute, with each value being uniquely identified by membership function $u$ in these fuzzy partitions.

\section{Algorithm for fuzzy partition generation}

1. read fuzziness parameter $\mathrm{m}$

2. for each $\mathrm{q}_{\mathrm{p}} \in \mathrm{QA}(\mathrm{p}=1,2,, \ldots, \mathrm{r})$

3. $\mathrm{FPp}=$ apply_ROI $\left(\mathrm{q}_{\mathrm{p}}\right)$

4. for each partition $\mathrm{t} \mathrm{FPi}$

5. label $t$ appropriately

6. function apply_ROI(q)

7. read $\mathrm{k}$ (number of ROI)

8. until $\max \left\{\left|\mu_{\mathrm{ij}}{ }^{(\mathrm{k}+1)}-\mu_{\mathrm{ij}}{ }^{(\mathrm{k})}\right|\right\}<\delta$

9. for each $x_{i} \in D(i=1,2, \ldots, N)$

10. for each ROI $j(j=1,2, \ldots, C)$

11. calculate $\mu_{\mathrm{ij}}$

12. $\mathrm{FP}=$ set of fuzzy partitions after completion

of above iteration

13. return FP

This process is repeated for each attribute till fuzzy partitions for each one of them is obtained. The NFARM measures for defining t-norm and cardinality of fuzzy set in finite universe $\mathrm{D}$ is given by the following equation (1) and (2) [4], [5],[7]. Table 4.2 explains about the t-norms of fuzzy sets. Fuzzy sets A and B in $\mathrm{D}$ are mapped as $\mathrm{D} \rightarrow[0,1]$ with $\mathrm{A}(\mathrm{x})$ and $\mathrm{B}(\mathrm{x})$ being the degrees to which attributes $\mathrm{A}$ and $\mathrm{B}$ are present in a transaction dataset $\mathrm{x}$ respectively.

$$
\mathrm{A} \cap \mathrm{T} \mathrm{B}(\mathrm{x})=\mathrm{T}(\mathrm{A}(\mathrm{x}), \mathrm{B}(\mathrm{x}))
$$

$$
|\mathrm{A}|=\Sigma_{(\mathrm{x} \in \mathrm{D})} \mathrm{A}(\mathrm{x})
$$

$\operatorname{supp}(\mathrm{A} \Rightarrow \mathrm{B})=\Sigma_{(\mathrm{x} \in \mathrm{D})}\left(\mathrm{A} \cap_{\mathrm{T}} \mathrm{B}\right)(\mathrm{x}) /|\mathrm{X}|$

$$
\operatorname{conf}(\mathrm{A} \Rightarrow \mathrm{B})=\Sigma_{(\mathrm{x} \in \mathrm{D})}\left(\mathrm{A} \cap_{\mathrm{T}} \mathrm{B}\right)(\mathrm{x}) / \Sigma(\mathrm{x} \in \mathrm{D}) \mathrm{A}(\mathrm{x})
$$

Table 4.2. t-norms in fuzzy sets

\begin{tabular}{|c|}
\hline t-norm \\
\hline$T_{M}(x, y)=\min (x, y)$ \\
\hline$T_{P}(x, y)=x y$ \\
\hline$T_{W}(x, y)=\max (x+y-1,0)$ \\
\hline
\end{tabular}

\subsection{Fuzzy Association Rule Generation}

The algorithm used in this paper consists of partition approach to generate novel fuzzy association rules. Depending on the ROI values, objects are partitioned with their respective attribute value. From these partitioned values ones which are frequent over the whole dataset $\mathrm{E}$ are made output. Then for each remaining attribute value, identify the constituent singletons $S_{1}$, $\mathrm{S}_{2}, \ldots \ldots \mathrm{S}_{\mathrm{m}}$ and then obtain the tid list of it $\mathrm{td}[\mathrm{it}]$ by intersecting the tid lists of all the constituent singletons. Additionally, the count of each singleton it is updated in count [it]( fig lines 610). Thus, an alternate between outputting and deleting itemsets, and creating tid lists for itemsets until no more itemsets are left. Thus, the algorithm terminates. ( figs lines 1-12)

\section{Pseudo-Code for forming NFARM Rules}

1) traverse each partition $P$

2) for each itemset it to $P$ in $1^{\text {st }}$ phase

3 ) if it is frequent(based on count $[i t]$ ) over the whole database $\mathrm{E}$

4) $\operatorname{output}(i t)$

5) remove $i t$

6) for each remaining itemset it

7) identify constituent singletons $\mathrm{s}_{1}, \mathrm{~s}_{2}, \ldots, \mathrm{s}_{\mathrm{m}}$ of it $\forall$ it $=\mathrm{s}_{1}$ $\cap s 2 \ldots . \cap s \mathrm{~m}$

8) tidlist $\operatorname{td}[i t]=$ intersect tidlists of all constituent singletons

9) calculate $\mu$ for it using $\operatorname{td}[\mathrm{i} \mu \mathrm{t}]$

10) $\operatorname{count}[i t]+=\mu$

11) if no itemsets remain to be enumerated

12) exit

\section{EXPERIMENTAL RESULTS}

In the test phase the new test image that has to be diagnosed is taken and also the techniques that are applied in the training phase like pre-processing and feature extraction are also applied in the new test image. From the extracted value the rules are mined and it is compared with the classified rules obtained in the training phase. The Rules that are generated in the training phase are stored in the transactional database, that contains the objects with their corresponding attribute values which are generated from the novel fuzzy association rule mining approach. Depending on the frequency of the itemset they are classified into normal, benign and malignant cells. Figure 5.1 represents the classified tumor cell.

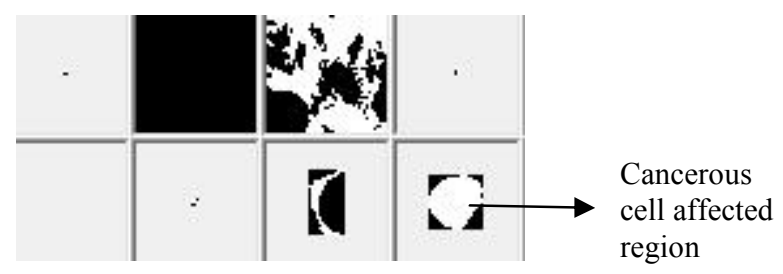

Figure 5.1 Features extracted from the segmented object holding tumor cell 
The objects attribute value in each ROI differs in its pixel value. The most frequent pixel values represent the normal tissues and the pixels which are singled out contains another two different pixel values. The performance metric is the total execution time needed for the mining operation handled by the proposed algorithm. The execution time is 9-20 times faster than fuzzy apriori depending on the support value used. More importantly, for the itemset, there is a particular support value for which optimal number of itemsets are generated. The support value for the proposed system is less than the support value of fuzzy apriori algorithm. The proposed algorithm performs most efficiently and speedily at its optimal support value which occurs in the range of $0.15-0.2$ for the itemset. For the support values, the number of classifications is as close to 1 as possible. Figure 5.2 illustrates the efficiency of the proposed system with respect to support and time value. The support value ranges from 0.075 to 0.4 in the $\mathrm{x}$-axis and the time taken for the mining process in microseconds in the y-axis. Hence the experimental results shows that the NFARM algorithm takes less execution time compared to the Fuzzy apriori algorithm.

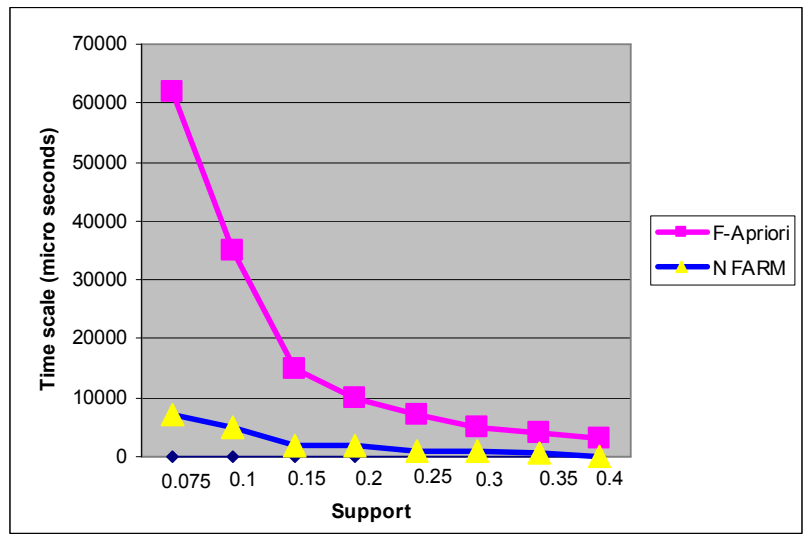

Figure 5.2 Illustrates the efficiency of NFARM compared with Fuzzy Apriori Algorithm

\section{CONCLUSION}

In the proposed system, the CT scan brain images are taken and the unwanted information that is inconsistent to the proposed work is eliminated using the pre-processing technique. From the pruned images the Regions of Interest are extracted and the attribute values are derived for detected Regions of Interest (ROI). The fuzzy partitioning algorithm is applied on the attributes that are extracted from the ROI and on applying the NFARM algorithm, to identify the cancerous cells. The results derived from this technique provide better efficiency of the proposed Novel Fuzzy Association Rule Mining algorithm compared to the traditional Fuzzy Apriori algorithm. The proposed method helps the physicians for making better decision by providing the keywords and identifying the cancerous cells.

\section{REFERENCES}

[1] Serhat Ozekes.,A.Yilmez Camurc :Computer aided detection of Mammographic masses on CAD digital Mammograms.: stanbul Ticaret Üniversitesi Fen Bilimleri (2005) pp.87-97

[2] Ruchaneewan Susomboon, Daniela Stan Raicu, Jacob Furst.:Pixel - Based Texture Classification of Tissues in computed Tomography.: Literature review (2007)

[3] De Cock, M.Cornelos, C.Kerre E.E. : Fuzzy Association Rules : A Two - sided Approach In: FIP, PP 385390(2003)

[4] Yan, p, Chen, G, Corneils, C,De Cock, M, Kerre, E.E: Mining Positive and Negative Fuzzy Association Rules In : KES, PP 270-276 Springes (2004)

[5] Verlincle, H, De Cock, M, Boute, R: Fuzzy Versus Quantitative Association Rules : A Fair Data- Driven Comparision: IEEE Transactions on Systems, Man, and Cybernetics- Part B : Cybernetics, 36,679-683 (2006)

[6] Agrwal. R., Imielinsk., T, Swam., A.N. : Mining Association Rules between sets of Items in Large Databases.:. S/G Mod Record 22, 207-216(1993)

[7] Laila Elfangary and Walid Adly Atteya: Mining MedicalDatabases using Proposed Incremental Association Rules Algorithm (PIA).: Second International Conference on the Digital Society ,IEEE Computer Society(2008)

[8] Pudi. V., Harilsa., j. : On the optimality of association rule mining algorithms. Technical Report TR-2001-01, DSL, Indian Institute of Science (2001)

[9] Hoppner. F., Klawonn. F.; Kruse, R, Rurkler, T.: Fuzzy cluster Analysis, methods for classification Data Analysis and Image recognition.: Wiley, New York (1999)

[10] Delgado. M., Marin. N., Sanchez. D., Vila. M.A. : Fuzzy Association Rules : General Model and Applications. IEEE Transaction of Fuzzy systems 11, 214-225(2003)

[11] Shu-yue. J., Tsung. E., Yenng. D., Daming. S.: Mining Fuzzy association rules with weighted items In: IEEE International Conference on SMC, pp 1906-1911, IEEE(2000)

[12] Cheng., Yan. P., Kerre. E.E.: Computationally Efficeint mining for Fuzzy Implication Based Association Rules in Quantitative Database.: International Journal of General systems, 33, 163-182(2004)

[13] J.S. Park, M. Chen, and P.S. Yu, "An Effective Hash Based Algorithm for Mining Association Rules," Proc. ACM IGMOD,(1995).

[14] Zaiane. O.R. Har .J.: Multimedia Miner: a system prototype for multimedia data mining.: , in : Proc. ACM- SIG MOD, Seattle, 1998, pp 581-583

[15] Wirth. M.D., Nikitenko.J. : Segmentation of the Breast Region in Mammograms using a Rule - Based Fuzzy 
Reasoning Algorithm.: ICGST-GVIP Journal, Volume 3 Issue on, Jan 2005

[16] C.Oronez, E. Omiecinski.; Discovering association rules based on image content, in: IEEE Advances in Digital Libraries Conference, 1999

[17] Wynne Hsu, MongLiLee, Ji Zhang, : Image Mining : trends and developments, Journal of Intelligent Information systems 9(1) (2002) 7-23.

[18] Maria-Luiza Antonie, Osmar R. Zaiane, Alexandra Coman, : Application of data mining techniques for medical image classification. In : Proc. Second Int. WorkShop on Multimedia Data Mining (MDM/KDD’2001)
[19] Tiawei Han and Micheline Kamber.: Data Mining Concepts \& techniques.: Morgan Kaufmann,2001

[20] Fafael C.Gonzalez and Richard E. Woods.: Digital Image Processing $2^{\text {nd }}$ edition. Addision Wesley 1993

[21] Hanchuan Peng, Fubui Long, and Chris Ding.: Feature Selection based on mutual information: Criteria of Maxdependency,Max_relerance and Min_redundancy.:IEEE Transaction on Pattern Analysis and machine Intelligence, Vol. 27 , No. 8, pp . 1226-1238,2005 